\title{
A Comparative Study of Three TDMA Digital Cellular Mobile Systems (GSM, IS-136 NA-TDMA and PDC) Based On Radio Aspect
}

\author{
Laishram Prabhakar \\ Manipur Institute of Management Studies \\ Manipur University \\ Canchipur, Manipur, India
}

\begin{abstract}
As mobile and personal communication services and networks involve providing seamless global roaming and improve quality of service to its users, the role of such network for numbering and identification and quality of service will become increasingly important, and well defined. All these will enhance performance for the present as well as future mobile and personal communication network, provide national management function in mobile communication network and provide national and international roaming. Moreover, these require standardized subscriber and identities. To meet these demands, mobile computing would use standard networks. Thus, in this study the researcher attempts to highlight a comparative picture of the three standard digital cellular mobile communication systems: (i) Global System for Mobile (GSM) -- The European Time Division Multiple Access (TDMA) Digital Cellular Standard, (ii) Interim Standard-136 (IS-136) -- The North American TDMA Digital Cellular Standard (D-AMPS), and (iii) Personal Digital Cellular (PDC) -- The Japanese TDMA Digital Cellular Standard.
\end{abstract}

Keywords-Comparative Study; GSM; IS-136 TDMA; PDC; Radio Aspect.

\section{INTRODUCTION}

In the last two centuries, mobility has been redefined such that both physical and virtual object are now mobile. The foundation of mobility of information was laid by Joseph Henery (1797-1878) who invented the techniques for distant communication. Later Samuel F.B Morse used the same property of electricity to invent the Telegraph. In 1876, Alexander Graham Bell laid the foundation by making the first voice call over wire i.e. "Mr. Watson, come here, I want to see you."

After the launch of Sputnik by USSR in 1957, US formed Advance Research Project Agency (ARPA) and laid the foundation of packet switched data networks. With the evolution of computer and the packet switched network movements, now byte has move to a new state of maturity. The convergence of telecommunication and information technology in 1965 leads to Information Communication Technology (ICT) that addresses the need to accommodate data formation and knowledge from anywhere and anytime.

Due to the achievement and advancement of the ICT, mobile computing has become a very important part. Now it can be defined as a computing environment over physical mobility. In this environment, a user has the capacity to perform anywhere, using a computing device, in the public, corporate and personal information spaces. While on the move, the preferred device could be a mobile device, and back home or in the office, a desktop computer could be preferred. Nevertheless computing should be through wired and wireless media -- be it for the mobile workforce, holidaymakers, enterprises or rural population. The access to information and virtual objects through mobile computing are absolutely necessary for optimal use of resource and increased productivity. Thus, mobile computing is used in different contexts such as virtual home environment and nomadic computing.

\section{MOBILE COMMUNICATION SYSTEMS}

It has been known for centuries that knowledge is power but in this information age, communication is becoming the real power. In this present age, mobile communication takes a great role. For example, a modern aircraft with 800 seats already offer limited Internet access. However, aircraft of the next generation would offer easy Internet access. In this scenario, a mobile network moving at high speed above the ground with a wireless link will be the only means of transporting data to and from passengers. Again the underlying vision for the emerging mobile and personal communication service and the system is to enable communication any time, any place and in any form. Thus, for seamless communication, personal communication cover terminal mobility provided by wireless access, personal mobility based on personal number and service portability through use of intelligent network capabilities.

Terminal mobility systems are characterized by their ability to locate and identify a mobile terminal or it moves and allows the mobile terminal to access telecommunication services from any location and even while on the move. In this scenario, communication is always between the network and the static terminal. So call delivery and billing are always based on terminal identity and mobile station number.

In personal mobility, the relation between the terminal and the user is dynamic and the call delivery and billing can be based on a personal identity (personal no) assign to the user. This is characterized by the ability to identity end user on the move and allows end users to originate, receive calls, and access subscribed telecommunication services, in any location. It is applicable to both the wired and the wireless network. 
In service portability, it refers to the capability of a network provided subscribed services at the terminal or location designated by the user. It is accomplished through the use of IN concept whereby the user service profile can be maintained in a suitable data base and the user can access, interrogate and modify to manage and control subscribed services.

\section{Time Division MultiPle ACCESs Digital Cellular Mobile System}

\section{A. Global System for Mobile}

Development of GSM started in 1982 within European Conference of Postal and Telecommunications Administrations (CEPT) for a future pan-European Cellular system. This was designed to replace the incompatible analog systems. The development was transferred to European Telecommunications Standards Institute (ETSI) in 1989 and the phase 1 standards were frozen in 1990. The first commercial GSM service was launched in 1992 and) the first GSM-1800 is also called DCS (Digital Cellular Service) DCS1800 was launched in September 1993. GSM standardization continues with Phase 2 standards completed in 1995. The enhancement of GSM services from the original concept of a pan-European standard shows that GSM was an attractive option to operators around the world including USA, and has become the number one digital cellular standard.

\section{B. Interim Standard-136: North American Time Division Multiple Access}

North American TDMA, often referred as TDMA, was developed in response to the need to increase cellular capacity. Unlike Europe and Japan where additional spectrum was made available for second generation digital systems, US operators were constrained to re-use the same spectrum used for Advance Mobile Phone System (AMPS). As a result the TDMA standard was developed to be compatible with the analogue AMPS system. Again the pressure on capacity forced the Telecommunications Industry Association (TIA) to consider a rapid development of a digital standard. As a result two TDMA standards were developed. Interim Standard - 54 (IS-54) often referred to as Digital-AMPS (D-AMPS), was the first of these. It shares the same 21 analogue call set-up channels with AMPS so that the call processing is the same between the two systems and handsets can support dual AMPS/ D-AMPS. The second phase standard is IS-136 which implements digital call set-up channels to enable stand-alone TDMA handsets. IS-136 has effectively replaced IS-54.

\section{Personal Digital Cellular}

Personal Digital Cellular (PDC) is a second-generation technology used in digital cellular telephone communication in Japan. It uses a variation of TDMA which divides each cellular channel into individual time slots in order to increase the amount of data that can be carried. PDC is currently used only in Japan, with the first systems introduced by Nippon Telegraph and Telephone (NTT) DoCoMo in 1991 as a replacement for the earlier analog networks. It operates in the $800 \mathrm{MHz}$ and $1,500 \mathrm{MHz}$ bands, making very efficient use of the available bandwidth. With bandwidth demand so high in Japan, the system can operate in two modes: full rate and half rate. Half-rate channels have reduced speech quality and data transmission rates, but allow more channels to occupy the same bandwidth. Subscriber numbers are so high in Japan that, although PDC is only operational in this one country, it accounted for $12 \%$ of global digital subscriptions in December 1999.

Along with the other mobile communication standards, PDC can be developed along a gradual evolutionary path to the global International Mobile Telecommunications -2000 (IMT2000) standards. Indeed, one of the IMT-2000 technologies, Wideband Code Division Multiple Access (WCDMA), is going through initial testing in Japan.

\section{NEED OF THE STUDY}

As mobile and personal communication services and networks involve providing seamless global roaming and improve quality of service to its users, the role of such network aspect as numbering and identification and quality of service will become increasingly important and well defined. To provide national management function in mobile communication network and provide national and international roaming, well defined standardized subscriber and identifies are required. To meet these demands, mobile computing will use standard networks. Some of the standard digital cellular mobile communication systems are (i) Global System for Mobile (GSM) -- The European Time Division Multiple Access (TDMA) Digital Cellular Standard, (ii) IS-136 -- The North American TDMA Digital Cellular Standard (iii) Personal Digital Cellular (PDC) -- The Japanese TDMA Digital Cellular Standard and (iv) IS-95 -- The North American Code Division Multiple Access (CDMA) Digital Cellular Standard. The cellular industry continues to experience massive growth. While there remains a large subscriber base for analog systems, most of the recent growth has been on digital systems. So this is an attempt in trying to enhance knowledge of the networks by comparing the networks based on radio aspects.

\section{Objective Of The Study}

The main objective of this paper is to study the three TDMA based Cellular mobile system namely (i) GSM: The European TDMA Digital Cellular Standard, (ii) IS-136: The North American TDMA Digital Cellular Standard (D-AMPS) (iii) PDC: The Japanese TDMA Digital Cellular Standard and to prepare a comparative analysis based on the Radio Aspects.

\section{COMPARATIVE ANALYSIS}

The International Telecommunication Union (ITU), which manages the international allocation of radio spectrum, allocated the bands $890-915 \mathrm{MHz}$ for the uplink and 935-960 $\mathrm{MHz}$ for the downlink for mobile in Europe. Since this range was already used in the early 1980 s by the analog systems of the day, the CEPT had the foresight to reserve the top $10 \mathrm{MHz}$ of each band for the GSM network that was still being developed. Eventually, GSM will be allocated to the entire $2 \mathrm{x}$ $25 \mathrm{MHz}$ bandwidth.

On the other hand the radio technology used in the IS-136 system provides a channel for advance services and improved system efficiency through the use of voice digitization, speech compression (coding), efficient radio modulation, enhanced radio frequency $(\mathrm{RF})$ power control, and a flexible approach to 
spectrum usage. D-AMPS will utilize the currently allocated spectrum for analog AMPS that is a total of $50 \mathrm{MHz}$ (uplink) and $869-894 \mathrm{MHz}$ (downlink), with each frequency channel to $30 \mathrm{KHz}$ spacing. Each frequency channel then is timemultiplexed with a frame duration of 40ms, which is partitioned into six slots of $6.67 \mathrm{~ms}$ duration.

PDC is the most spectrally efficient of TDMA technologies, with six half-rate or three full-rates channels possible in a 25 $\mathrm{kHz}$ frequency space, compared to three channels in $30 \mathrm{kHz}$ in IS-136 and eight channels in $200 \mathrm{kHz}$ for GSM. It even compares favorably to Code Division Multiple Access (CDMA), using spread-spectrum technology to allow up to 131 channels in a $1,250 \mathrm{kHz}$ spectrum band.

Full-rate speech normally requires a digital data transfer rate of $9.6 \mathrm{kbps}$ (kilobits per second), as is used in GSM, and TDMA IS-136 networks. PDC offers two alternative rates: $9.6 \mathrm{kbps}$ in full-rate channels or $5.6 \mathrm{kbps}$ in the half-rate channel. The quality of speech along a $5.6 \mathrm{kbps}$ connection is significantly lower than the standard $9.6 \mathrm{kbps}$ connection, but is a useful trade-off with the number of channels available.

About the advantages of PDC, the newly developed Linearized Saturated Amplifier with Bidirectional Control (LSA-BC) improves efficiency. Although it is a saturated amplifier, the voltage controlled power supply results in linear operation. Coherent detection with Adaptive Carrier Tracking (ACT) has been developed for digital systems. ACT gives excellent performance under fast Rayleigh fading because of fast carrier tracking ability. ACT is made by all digital circuits so that adjustment are not necessary and power dissipation is very low (1/40 that of conventional differential demodulator). A direct modulator made of only one differential amplifier is advantageous in terms of compactness and power consumption (1/8 that of previously developed modulator IC's).

The comparison of three cellular digital systems are done through a number of sub parameters like downlink frequency, uplink frequency, symbol rate frequency channel, modulation, access etc. and shown in the table no.1.

It is seen that the number of channels/carriers of GSM is greater than IS-136 and PDC. It is also observed that PDC employs diversity reception in the mobile station which obviates the need for equalizers, which are an essential component of GSM. PDC uses a much lower transmission bit rate (42kb/sec vs. $270.83 \mathrm{~kb} / \mathrm{sec}$ in GSM/48.6kb/sec in IS-136) which leads to better spectrum utilization, higher capacity and lower cost. PDC has significant similarities with the IS-136 DAMPS system.

The call sequence of GSM is similar to that of IS-136. However, it uses gateway Mobile Switching Centre (MSC) or International Mobile Subscriber Identity (IMSI). The call sequence of IS-136 is similar to that of GSM but recently it does not use gateway MSC and IMSI. It uses mobile identification number (MIN). However, PDC uses gateway mobile control centre. The access signaling protocols in PDC are simpler and require fewer procedures and are simpler to use and lower in cost.
TABLE I. RADIO PARAMETER AND CHARACTERISTICS FOR GSM, IS-136 AND PDC

\begin{tabular}{|c|c|c|c|}
\hline Parameter & GSM & IS-136 & PDC \\
\hline $\begin{array}{l}\text { Downlink } \\
\text { frequency }(\mathrm{MHz})\end{array}$ & $\begin{array}{l}935-960 \\
1805-1880 \\
1930-1990\end{array}$ & $\begin{array}{l}869-894 \\
1930-1990\end{array}$ & $\begin{array}{l}810-826 \\
1429-1453\end{array}$ \\
\hline $\begin{array}{l}\text { Uplink } \\
\text { frequency }(\mathrm{MHz})\end{array}$ & $\begin{array}{l}890-915 \\
1710-1785 \\
1850-1910\end{array}$ & $\begin{array}{l}824-849 \\
1850-1910\end{array}$ & $\begin{array}{l}940-956 \\
1477-1501\end{array}$ \\
\hline Symbol rate & $\begin{array}{l}271 \mathrm{ksymbols} \\
(271 \mathrm{kbit} / \mathrm{s})\end{array}$ & $\begin{array}{l}24.3 \\
\text { ksymbols } \\
(48.6 \text { kbits/s) }\end{array}$ & $\begin{array}{l}21 \\
\text { ksymbols } \\
(42 \text { kbits/s) }\end{array}$ \\
\hline $\begin{array}{l}\text { Frequency } \\
\text { channels }\end{array}$ & $200 \mathrm{KHz}$ & $30 \mathrm{KHz}$ & $25 \mathrm{KHz}$ \\
\hline Modulation & $\operatorname{GMSK}^{*}(\mathrm{BT}=0.3)$ & $\begin{array}{l}\pi / 4 \quad-D^{-D P S K} \\
(\alpha=0.35)\end{array}$ & $\begin{array}{l}\pi / 4-\mathrm{DQPSK} \\
(\alpha=0.5)\end{array}$ \\
\hline Access & FH/TDMA & TDMA & TDMA \\
\hline $\begin{array}{l}\text { Channels/ } \\
\text { Carrier } \\
\end{array}$ & $\begin{array}{l}8 \text { Full Rate } \\
16 \text { Half Rate }\end{array}$ & $\begin{array}{l}3 \text { Full Rate } \\
6 \text { Half Rate }\end{array}$ & $\begin{array}{l}3 \text { Full Rate } \\
6 \text { Half Rate }\end{array}$ \\
\hline Voice Codec & $\begin{array}{l}\text { RPE-LTP*** } \\
22.8 \mathrm{~kb} / \mathrm{s} \text { full, } \\
13 \mathrm{~kb} / \mathrm{s} \\
\text { Source, VSELP } \\
11.4 \mathrm{~kb} / \mathrm{s} \text { half }\end{array}$ & \begin{tabular}{|l}
\multicolumn{2}{|l|}{ VSELP***** } \\
$13 \mathrm{~kb} / \mathrm{s} \quad$ full, \\
$7.95 \mathrm{~kb} / \mathrm{s}$ \\
source, $6.5 \mathrm{~kb} / \mathrm{s}$ \\
half
\end{tabular} & $\begin{array}{l}\text { VSELP } \\
11.2 \mathrm{~kb} / \mathrm{s} \text { full, } \\
6.7 \mathrm{~kb} / \mathrm{s} \text { Source, } \\
3.45 \mathrm{~kb} / \mathrm{s} \text { half } \\
\text { source }\end{array}$ \\
\hline Max delay time & $16 \mu \mathrm{s}$ & $50 \mu \mathrm{s}$ & $10 \mu \mathrm{s}$ \\
\hline
\end{tabular}

*GMSK: Gaussian Minimum Shift Keying

**DQPSK: Differential Quaternary Phase Shift Keying

***RPE-LTP: Regular Pulse Excitation with Long-Term Predictor

****VSELP: Vector Sum Excited Linear Prediction

\section{FINDING}

From the comparative analysis of the three standards the following are the highlights of the findings:

\section{A. GSM}

1) From the original concept of a pan-European standard, it soon became clear that GSM was an attractive option to operators around the world including USA, and has become the number one digital cellular standard.

2) Low terminal and service cost is an attractive feature for the users.

3) It has also an emergency service, where the nearest emergency service provider is notified by dialing three digits similar to 911.

4) Interestingly the key drivers for GSM are panEuropean roaming to offer compatibility throughout Europe and interaction with Integrated Services Digital Network (ISDN) and bill to home.

5) It has SIM (Subscriber Identity Module) card which contains the IMSI used to identify the subscriber to the system, a secret key for authentication, and other information. The International Mobile Equipment Identity (IMEI) and the International Mobile Subscriber Identity (IMSI) are independent, thereby allowing personal mobility. The SIM card may be protected against unauthorized used by a password or personal identity number. 
6) In GSM, Regular pulse excitation - long term prediction (RPE-LTP) scheme is employed in order to reduce the amount of data sent between the mobile station and base transceiver station. In essence, when a voltage level of a particular speech sample is quantified, the mobile station's internal logic predicts the voltage level for the next sample. When the next sample is quantified, the packet sent by the Mobile Station (MS) to the Base Transceiver Station (BTS) contains only the error (the signed difference between the actual and predicted level of the sample).

\section{B. $I S-136$}

1) It share the same 21 analog call set up channels with AMPS so that the call processing is the same between the two systems and handsets can support dual AMPS/D-AMPS.

2) Its modulation is $\pi / 4-D Q P S K$ with $(\alpha=0.35)$ so it provides lower envelopes fluctuation than standard DQPSK, allows non-coherent detection and performs well in a multipath environment.

3) The IS-136 system adds new power class of mobile phone to allow reduces the minimum cell site radius.

4) It supports a new function Mobile Assisted Channel Assignment (MACA) similar to Mobile Assisted Hand Over (MAHO). MACA is a process in which signal strength reporting takes place while mobile phone is monitoring a Digital Control Channel (DCCH) camping.

5) Each cell site in a cellular system has its own unique Digital verification color code (DVCC). A unique DVCC for each cell site ensures that the correct mobile phone is communicating with the proper station since frequencies are reused in most cellular systems.

6) It allows several types of phone identities. They are (a) TMSI - Temporary mobile station identity, (b) IMSI International mobile subscriber umber and (d) ESN Electronic serial number

7) Vector sum excited linear prediction (VSELP) is a speech coding method used in IS-136 (D-AMPS). It was used in the first version of RealAudio for audio over the Internet. DAMPS (IS-54 and IS-136) VSELP specifies an encoding of each $20 \mathrm{~ms}$ of speech into 159-bit frames, thus achieving a raw data rate of $7.95 \mathrm{kbit} / \mathrm{s}$. In an actual TDMA cell phone, the vocoder output is packaged with error correction and signaling information, resulting in an over-the-air data rate of $16.2 \mathrm{kbit} / \mathrm{s}$. For internet audio, each 159-bit frame is stored in 20 bytes, leaving 1 bit unused. The resulting file thus has a data rate of exactly $8 \mathrm{kbit} / \mathrm{s}$.

\section{C. $P D C$}

1) It is found from the study that PDC is one of the most spectrally efficient of TDMA technologies. It has six half-rate or three full-rates channels possible in a $25 \mathrm{kHz}$ frequency space, compared to three channels in $30 \mathrm{kHz}$ in IS-136 and eight channels in $200 \mathrm{kHz}$ for GSM.

2) PDC compares favorably to Code Division Multiple Access (CDMA), using spread-spectrum technology to allow up to 131 channels in a 1,250 kHz spectrum band.
3) PDC offers two alternative rates: 9.6kbps in full-rate channels or 5.6kbps in the half-rate channel. The quality of speech along a 5.6kbps connection is significantly lower than the standard 9.6kbps connection, but is a useful trade-off with the number of channels available.

4) The PDC network supports many advanced features in-line with the other second-generation technologies, such as text messaging and caller identification.

5) Utilizing its Intelligent Network (IN) capabilities, PDC also supports pre-paid calling, personal numbers, Universal Access Numbers, advanced charging schemes and wireless virtual private networks (VPNs).

6) As already mentioned PDC is a TDMA system and it operates by splitting each channel into several time slots and thereby allowing several users to use the same frequency channel. For each channel it is possible to support three users under normal circumstances. However when traffic levels are high it is possible to use half data rate speech. Although this reduces the speech quality, it enables six calls to be supported by each channel. This compares very favourably to GSM that manages eight within each $200 \mathrm{kHz}$ channel.

7) Speech encoding is an important factor. PDC uses a different encoder to that used on IS54/IS136. The standard rate is 9.6 kbps along with similar technologies such as GSM, but when half rate encoding is used this falls to $5.6 \mathrm{kbps}$. Although this gives a significant reduction in voice quality, it is still adequate to maintain intelligibility and enables the network capacity to be increased to accommodate further calls.

8) It is also found that PDC has similar speech coding method like IS-136.

\section{CONCLUSION}

In this paper the researcher gives an overview of mobile communication and the standards of digital cellular especially for GSM: The European TDMA digital cellular standard, IS136: TDMA based digital cellular system in United States and PDC: The Japanese TDMA based digital cellular system. Nowadays industry speeding up the development of mobile communication system where both voice and data services can be delivered regardless of location, network or terminal. The study clears that the three digital cellular systems have their own special features that satisfy diverse needs of mobile commutation system.

\section{REFERENCE}

[1] A Sokeke, K Talukda, "A text Book on Mobile Computing" Tata McGraw Hill, 2005

[2] J. Schiller, "Mobile Communications" Pearson Education Limited, $3^{\text {rd }}$ Edition, 2006

[3] M. Mouly, M.B. Pautet, "The GSM System for Mobile Communications", published by M.Mouly et Marie-B. Pautet, Palaiseu, France, 1992

[4] N. Spencer, "An Overview of Digital Telephony Standards", 1998 The Institution of Electrical Engineers . 1.1 Printed and Published by IEE, Savoy Place, London WCPR OBL, UK.

[5] N. Nakajima, M Kuramoto and K. Kinoshita, "Development of a Digital Cellular Systems Using TDMA Technique", NIT Radio Communication Systems Laboratories, IT Mobile Communications Division, 1-2356 Take. Yokosukashi, 238-03 Japan 
[6] R. Ganesh and K. Pahlavan, "Wireless Network Deployments", Kluwer Academic Publishers, 2000

[7] R. Pandya, "Mobile and Personal Communication Systems and Services", Prentice Hall of India, $4^{\text {th }}$ Indian Reprint 2003

[8] S. Sampei, "Application of Digital Wireless Technologies to Global
Wireless Communication”, Prentice Hall PTR, 1997

[9] William C.Y. Lee, "Mobile Cellular Telecommunication - Analog and Digital Systems", MCGraw Hill International Edition, 1995.

[10] Yi_Bing Lin, Imrich Chlamtac, "Wireless and Mobile Network Architecture", Wiley, 2002 